\title{
Electronic Trial Master File Code
}

National Cancer Institute

\section{Source}

National Cancer Institute. electronic Trial Master File Code. NCI Thesaurus. Code C114460.

The symbol assigned to terms in the eTMF Content Model. 